\title{
Bilateral Ovarian Torsion: A Rare Complication of Ovarian Stimulation in In Vitro Fertilization
}

\author{
${ }^{1}$ Sonal Agarwal, ${ }^{2}$ Arya Rajendran, ${ }^{3}$ Haritha Mannem, ${ }^{4}$ Kamini A Rao
}

\section{ABSTRACT}

Aim: To enlighten about one of the rare complications of ovarian stimulation during in vitro Fertilization (IVF) and how to manage it with early diagnosis and intervention.

Background: Torsion of ovary, tube or both are estimated to be responsible for a minimal number of overall gynecological emergencies with an incidence of $2.7-7.4 \%$. It is considered a common diagnostic challenge in any emergency setting. Torsion should be referred and managed at a tertiary health center with all emergency facilities. The trending availability of assisted reproductive technology and its increasing success day by day has resulted in an increase in ovulation induction and its complications including OHSS and ovarian torsion in the present scenario.

Case report: At our center, one case of bilateral ovarian torsion as a consequence of controlled ovarian stimulation in a woman with PCOS was reported. This can be considered as a potentially fatal complication of pharmacological stimulation of ovary in assisted reproduction. Life-saving emergency laparoscopic surgery was undertaken. Timely diagnosis and intervention prevented oophorectomy and fertility was preserved.

Conclusion: An Early and prompt intervention to preserve ovarian function and conduct a fertility-conserving surgery should be laparoscopic whenever and wherever possible. Detorsion should be the treatment of choice in reproductive age women whose families are incomplete, regardless of the fact of the color of the ovary. Oophoropexy may be considered to prevent recurrence. In older women whose family are complete and in postmenopausal women, oophorectomy is the treatment of choice to remove the risk of retorsion.

Clinical significance: If on laparoscopy, a cyst whether nonfunctional or not is found in the ovary or paraovarian structure, cystectomy or interval cystectomy should be performed in younger women. Ovarian mutilating surgery is not considered to be a viable option in the management of torsion in a patient who is undergoing fertility preserving management except in cases with complete tissue necrosis.

Keywords: Bilateral ovarian torsion, Detorsion, Laparoscopy.

How to cite this article: Agarwal S, Rajendran A, Mannem $\mathrm{H}$, Rao KA. Bilateral Ovarian Torsion: A Rare Complication of

\footnotetext{
${ }^{1}$ FNB Fellow, ${ }^{2,3}$ Fellow, ${ }^{4}$ Medical Director

${ }^{1-4}$ Department of Reproductive Medicine, Milann Fertility Centre, Bangaluru, Karnataka, India
}

Corresponding Author:Sonal Agarwal, FNB Fellow, Department of Reproductive Medicine, Milann Fertility Centre, Bangaluru, Karnataka, India, e-mail: sonaljaipur28@gmail.com
Ovarian Stimulation in In Vitro Fertilization. Int J Infertil Fetal Med 2018;9(1\&2):22-24.

Source of support: Nil

Conflict of interest: None

\section{BACKGROUND}

Individualization of $\mathrm{COH}$ regimen and pro-active diagnosis and intervention in case of any complication is the key to success in saving a life. In a patient of PCOS and with a history of OHSS, proper workout and discussion on $\mathrm{COH}$ protocol before starting so as to prevent hyperstimulation of ovaries is significantly essential. Hyperstimulated ovaries presenting with acute pain abdomen ${ }^{1}$ are often misdiagnosed at start and torsion is thus reported to be in the range of $0.08-0.2 \% .^{2}$ Surgical intervention to maintain integrity and functionality of ovarian reserve is tough and very challenging due to friability of tissue. Assessment of degree of ischemia is utmost important in deciding for the further plan of management.

\section{CASE REPORT}

The 32-year-old lady married since 13 years, irregular menstrual cycles, known case of PCOS presented with secondary infertility of 7 years duration. She had one living child of 8 years of age which was an IUI conception. Her past medical history revealed three cycles of ovulation induction and intrauterine insemination but failed to conceive. She had a history of moderate OHSS during LBG protocol of controlled ovarian stimulation for IVF in 2012. It was managed conservatively. She did not conceive in further embryo transfer cycles. When she presented again for IVF-ICSI in March 2017, flexible antagonist protocol was started after looking into her hormone levels for ovarian reserve. The total dose of gonadotropins given was 3025 IU. Agonist trigger was given and the patient posted for egg retrieval after 36 hours. Ovum pickup were done at 10 am on 15/04/2017. Nine oocytes were retrieved. Elective cryopreservation done because of the previous history of OHSS and poor endometrium. The patient was sent home after 4 hours with instructions to take plenty of fluids, restricted physical activity, 


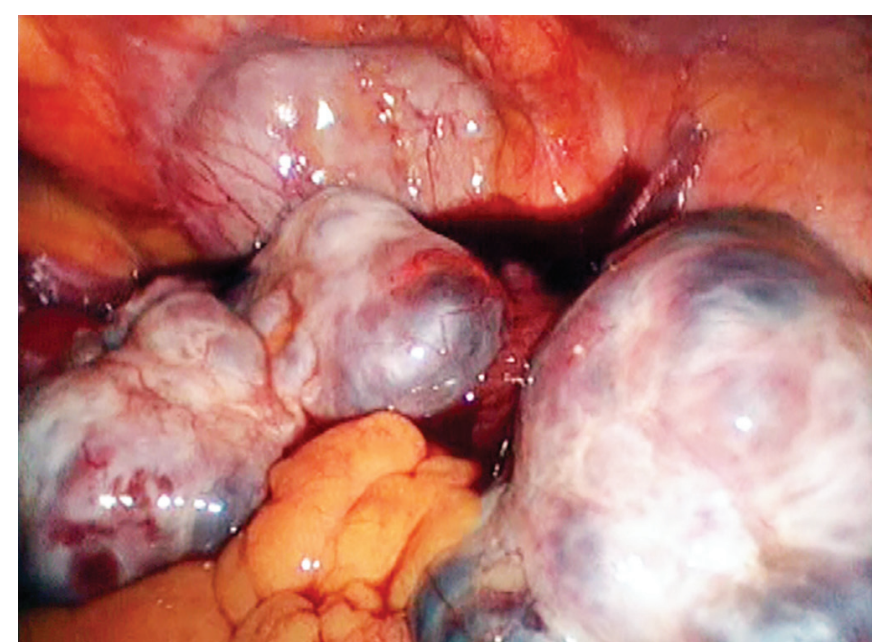

Fig. 1: Laparoscopic view of pelvis showing enlarged and oedematous fallopian tubes and ovaries

abstinence and protein-rich diet. After 3 hours of discharge, the patient returned with complaints of severe abdominal pain and vomiting.

We suspected OHSS at first and admitted the patient for conservative management. She was treated symptomatically for 5 hours with fluids, plasma expanders (human albumin $20 \%$ ) and antiemetics, c-tide antagonist and cabergoline tablet.

The patient was intimated about the complications and asked to restrain her movements in the ward. Vital monitoring was done continuously. Blood investigations like hematocrit, renal function tests, liver function tests, and electrolytes done. All were within normal limits. The transabdominal scan showed fluid in pelvic fossa and internal echoes consistent with the appearance of blood clots suggesting hemoperitoneum. There was a deterioration of symptoms till evening, and therefore scan was repeated for her. On Doppler there was decreased flow around ovaries and "whirlpool sign" of twisting of fallopian tubes was noted.

Bilateral ovarian torsion ${ }^{3}$ was suspected, and patient and attendants explained and counseled for laparoscopic exploration. Emergency laparoscopy was done in view of acute abdominal pain and ovarian torsion.

The $10 \mathrm{~mm}$ trocar was placed on the left side, and secondary trocars were inserted at opposite sites, one in the right upper abdominal quadrant and the other on the extreme left of the middle abdominal quadrant (Fig. 1). These secondary trocars were inserted under direct visual control from the primary trocar. Right ovarian pedicle had three and a half clockwise turn on itself and left ovary had one and a half anti-clockwise turn. Both tubes were oedematous. Two atraumatic probes were introduced through secondary ports for irrigation and suction and handling of uterus and ad-

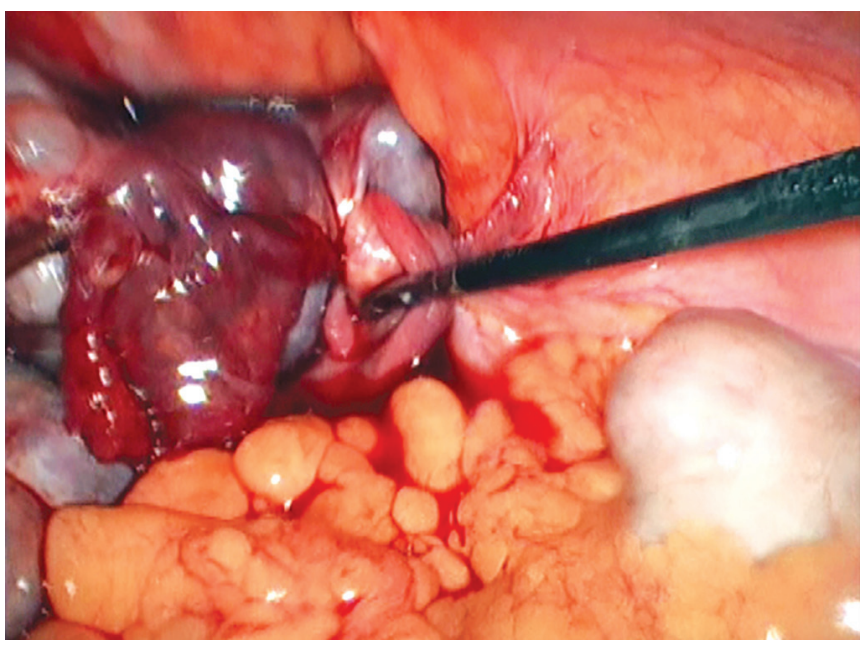

Fig. 2: Laparoscopic picture demonstrating detorsion of right ovary

nexa. One atraumatic probe was used to elevate twisted adnexa, pushing it contralaterally in the direction of rotation. ${ }^{4}$ Serial manipulations were done to achieve unwinding of the adnexa. Suction and irrigation have done. Bilateral pedicles of ovary and tube were carefully examined for improvement in color and decrease in edema (Fig. 2). The beginning of recovery was seen as adnexa turned pink in color shortly after the manipulation. Multiple cysts were present, which were punctured with monopolar cautery. The drain was kept for 3 days. The patient was discharged with an uneventful postoperative period.

\section{DISCUSSION}

In this case, timely intervention by bilateral detorsion with an ovarian puncture was performed. Hemostasis was achieved. Thus ovaries were salvaged. At many places, oophorectomy is performed for adnexal torsion which has a negative impact on fertility of women. ${ }^{5}$ Ethically detorsion is a valid and more conservative approach. In cases with a high probability of ovarian torsion, one should not wait in fear of negative findings on laparoscopy and should be prompt with surgery to restore ovarian blood supply before significant damage occurs. In such cases, a laparoscopic approach should be preferred to reduce postoperative pain and hospital stay. ${ }^{6}$ The route of surgery is completely dependent on the surgeon's expertise and suitability.

\section{CONCLUSION}

Ovarian torsion is far less common than other causes of an acute abdomen. Diagnosis relies on detailed history and clinical findings collaborating along with ultrasound 
findings with keeping torsion in mind with suspicion. Diagnosing torsion in enlarged ovaries is tremendously difficult on clinical perspective. Ultrasound along with Doppler aids in the diagnosis. Abnormal Doppler signals are found in complete cases of torsion, but the complete absence of perfusion of the ovary may be a late sign. So the presence of vascular flow does not exclude torsion as a diagnosis. Findings depend on the pathophysiology of adnexa dynamically. Detorsion followed by oophoropexy, ${ }^{7}$ rather than oophorectomy, are surgical techniques that are the recent trends of medicine and are gaining popularity. Newer technologies like shortening of utero ovarian ligament are being considered for future practice. The cause of bilateral ovarian torsion is still a dilemma, and the main emphasis should be on careful handling of the patient with these enlarged friable ovaries to avoid catastrophe.

\section{REFERENCES}

1. Anteby SO, Schenker JG, Polishuk WZ. The value of laparoscopy in acute pelvic pain. Ann Surg 1975;181(4):484-486.

2. Munshi S, Patel A, Banker M, Patel P. Laparoscopic detorsion for bilateral ovarian torsion in a singleton pregnancy with spontaneous ovarian hyperstimulation syndrome. J Hum Reprod Sci. 2014;1:66-68.

3. Damigos E, Johns J, Ross J. An update on the diagnosis and management of ovarian torsion. The Obstetrician \& Gynaecologist 2012;14:229-236.

4. Lele PR, Tangri MK. A case of ovarian hyperstimulation syndrome with spontaneous hemoperitoneum. Int J Reprod Contracept Obstet Gynecol. 2017 Feb;6(2):766-768.

5. Hibbard LT. Adnexal torsion. Am J Obstet Gynecol 1985;152:456-461.

6. Hertzberg BS, Kliewer MA, Paulson EK. Ovarian cyst rupture causing hemoperitoneum: imaging features and the potential for misdiagnosis. Abdominal imaging. 1999 May 1;24(3):304-308.

7. Al Omari W, Ghazal-Aswad S, Sidky IH, Al Bassam MK. Ovarian salvage in bilaterally complicated severe ovarian hyperstimulation syndrome. Fertil Steril. 2011;96(2):e77-79. 